\title{
Static Gravitational Fields at Finite Temperature
}

\author{
F. T. Brandt* \\ Instituto de Física \\ Universidade de São Paulo \\ E-mail: fbrandteusp.br \\ J. B. Siqueira \\ Instituto de Física \\ Universidade de São Paulo \\ E-mail: joao@fma.if.usp.br
}

\begin{abstract}
The thermal effective Lagrangian of a static background of gravitational fields is derived in the high temperature limit. In the one-loop approximation we obtain a closed form result as a simple functional of the static metric tensor. At the two-loop order, we are able to compute the effective Lagrangian up to the contribution of the graviton self-energy. This encodes all the effects of selfinteractions of the thermal fields and may be employed in order to obtain the thermodynamical properties in a gravitational background.
\end{abstract}

36th International Conference on High Energy Physics,

July 4-11, 2012

Melbourne, Australia

${ }^{*}$ Speaker. 


\section{One-loop Static Thermal Effective Action}

When the temperature $T$ is non-zero, the one-loop effective Lagrangian for a gravitational background field can be formulated in terms of the following trace of the Laplace-Beltrami operator (here the relation $\log \operatorname{Det} A=\operatorname{Tr} \log A$ is being employed in the context of the imaginary time formalism $[1,2])$

$$
\mathscr{L}=-\frac{T}{2} \sum_{n} \int \frac{d^{d-1} p}{(2 \pi)^{d-1}} \log \left(-\beta^{2} p_{\mu} \tilde{g}^{\mu v} p_{v}\right) ; \tilde{g}^{\mu v} \equiv \sqrt{-g} g^{\mu \nu},
$$

where the Matsubara frequencies are such that $p_{0}=i 2 \pi n T$ (for simplicity, we are considering the example of thermal scalar fields; this can be generalized for spinor or vector fields in a straightforward manner). For static gravitational fields, in the high-temperature limit, when $T>>\vec{\partial} g$, the space dependence of the metric can be neglected, so that (see [3] and [4] for details)

$$
\mathscr{L}^{\text {stat. }}=-\frac{T}{2} \sum_{n} \int \frac{d^{d-1} p}{(2 \pi)^{d-1}} \log \left[-\beta^{2}\left(g^{00} p_{0}^{2}+2 g^{0 i} p_{0} p_{i}+g^{i j} p_{i} p_{j}\right)\right]+\ldots,
$$

where ... represents terms which are independent of $T$.

Let us now perform the change of variables

$$
p_{i} \rightarrow p_{i}^{\prime}=M_{i}^{j} p_{j}+f_{i} p_{0},
$$

where $M$ is symmetric. Upon imposing the condition

$$
p_{i}^{\prime} p_{i}^{\prime}=g^{i j} p_{i} p_{j}+2 g^{0 i} p_{0} p_{i}+f_{i} f_{i} p_{0}^{2}
$$

we obtain

$$
\left\{\begin{array}{l}
M_{i}^{j} M_{i}^{k}=g^{j k} \\
f^{i} M^{i j}=g^{0 j}
\end{array} .\right.
$$

Therefore, the effective Lagrangian can be written as

$$
\mathscr{L}^{\text {stat. }}=-\frac{T}{2} \frac{1}{\sqrt{-\operatorname{det}}} \sum_{n} \int \frac{d^{d-1} p^{\prime}}{(2 \pi)^{d-1}} \log \left[-\beta^{2}\left(\left(g^{00}-f^{i} f^{i}\right) p_{0}^{2}-p_{i}^{\prime} p_{j}^{\prime}\right)\right]
$$

where the entries of the matrix $\mathbf{g}$ are $g^{i j}$. Performing the transformation

$$
p_{i} \rightarrow \sqrt{g^{00}-f^{j} f^{j}} p_{i}
$$

we readily obtain

$$
\mathscr{L}^{\text {stat. }}=\mathscr{L}^{(0)} \frac{\left(g^{00}-\left(\mathbf{g}^{-1}\right)^{i j} g^{0 i} g^{0 j}\right)^{\frac{d-1}{2}}}{\sqrt{-\operatorname{det} \mathbf{g}}},
$$

where

$$
\mathscr{L}^{(0)}=\frac{\Gamma[d] \zeta(d)}{2^{d-2} \pi^{(d-1) / 2} \Gamma\left(\frac{d-1}{2}\right)(d-1)} T^{d}
$$

is the pressure of a free Boson gas in $d$ space-time dimensions. We can also express this result in terms of the co-variant metric components. Using the identity (this follows from $g^{\mu \alpha} g_{\alpha v}=\delta_{v}^{\mu}$ )

$$
g^{00}-\left(\mathbf{g}^{-1}\right)^{i j} g^{0 i} g^{0 j}=\left(g_{00}\right)^{-1},
$$


Eq. (1.8) can be written as

$$
\mathscr{L}^{\text {stat. }}=\mathscr{L}^{(0)} \frac{\sqrt{-g_{00} \operatorname{det} \mathbf{g}^{-1}}}{\left(g_{00}\right)^{d / 2}},
$$

Expanding the determinant of $g^{\mu v}$ in terms of co-factors and using the identity $g_{i 0}=-\mathbf{g}_{i j}^{-1} g^{j 0} g_{00}$ as well as (1.10) we can show that

$$
g_{00} \operatorname{det} \mathbf{g}^{-1}=\frac{1}{\operatorname{det} g^{\mu \nu}}=g .
$$

Therefore, Eq. (1.11) yields

$$
\mathscr{L}^{\text {stat. }}=\mathscr{L}^{(0)} \frac{\sqrt{-g}}{\left(g_{00}\right)^{d / 2}},
$$

which is in agreement with the known result when $d=4$ [5]. This static effective Lagrangian has been obtained previously using a much more involved approach in terms of the heat-kernel technique restricted to a static space-time, in a reference frame such that $g_{0 i}=0$ [6]. Since the heat bath breaks the invariance under general coordinate transformations, as it is evident due to the presence of the Matsubara sum in Eq. (1.1), it is essential to perform the calculation for general values of $g_{0 i}$. Physically one must impose that the heat bath is freely moving in a time-like geodesic, so that in the heat bath frame $g_{0 i}$ vanishes only in very special cases, even for static space-times.

\section{Higher loop orders}

For the special case of a static metric, the one-loop result shows that it is possible to sum all the 1PI one-loop $n$-graviton functions in terms of the closed form expression in Eq. (1.13). A key ingredient in order to obtain this result was the use the identity between the high temperature static and the zero momentum limit of all the one-loop thermal Green's functions (SZM for short). This property has been show to be true for other background fields, by explicitly computing the two- and three-point functions at one-loop order [7] (more recently we have employed an iterative procedure in order to prove the SZM identity for all one-loop 1PI amplitudes [4] ).

From the one-loop result one can obtain the thermodynamical properties, such as the pressure of non-interacting thermal particles subjected to an external gravitational field. However, in a more realistic physical scenario one would have to take into account the self-interactions of the thermal particles. In principle this can be investigated computing the higher loop contributions; these necessarily involves thermal field interactions. In order to tackle this issue in a systematic fashion, we first consider the possibility that the SZM identity holds also at the two-loop order for all the 1PI diagrams. This issue is also of interest from a broader point of view, since there are few higher loop results at finite temperature and our analysis is completely general to the extent that it includes theories with cubic and quartic vertices as well as more general cases, like gravity.

Here we present preliminary results which shows that the two-loop contributions to the selfenergy satisfy the SZM identity. We have considered all the non-trivial diagrammatic topologies which, unlike the ones shown in figures 1 and 2 are dependent on the external momentum and cannot be reduced to the one-loop case.

These are given by the five topologies shown in figure 3 (notice that we are taking into account the possibility of having vertices with any number of lines, as it would be in gravity). 

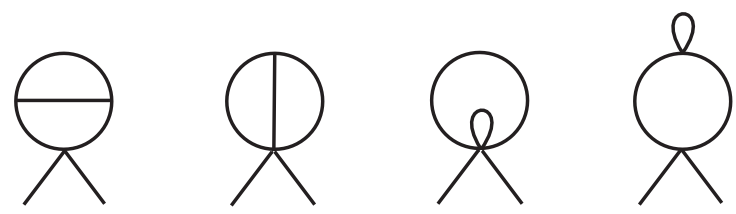

Figure 1: Momentum independent contribution to the two-point function at the two-loop order.

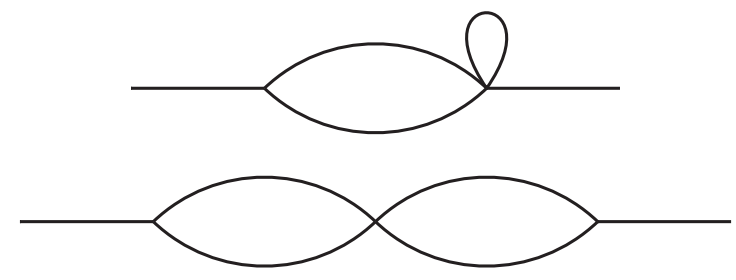

Figure 2: Diagrammatic topologies which can be reduced to the one-loop case.
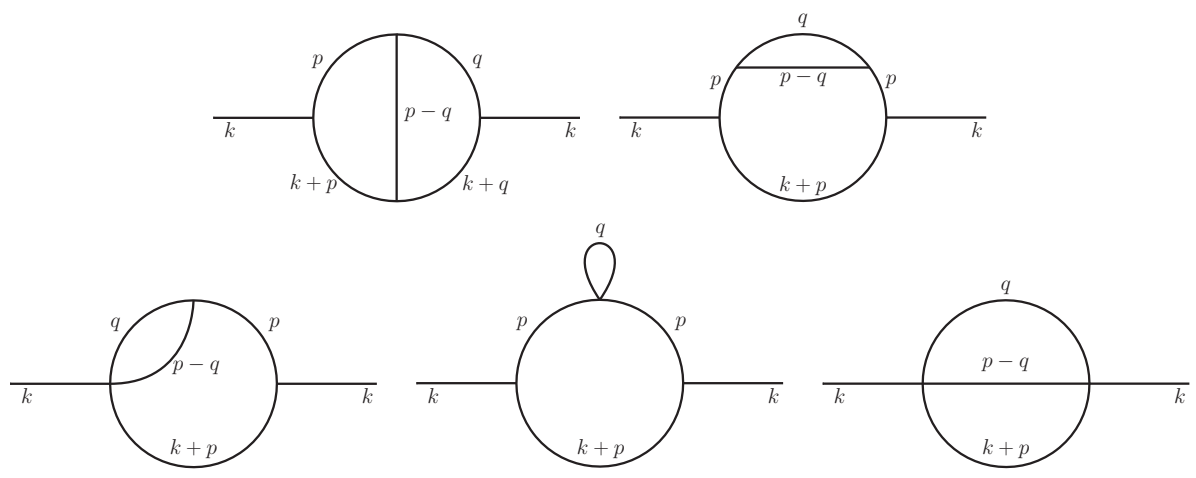

Figure 3: Non-trivial diagramatic topologies topologies.

When the external fields are Bosonic, we can consider the static limit before making the analytic continuation of the external energies. Using this, we have performed the Matsubara sum of these diagrammatic topologies for general interaction vertices, in $d$ space-time dimensions in both static and zero momenta limit. Our explicit results show that the SZM identity, as previously defined in the one-loop analysis, is also valid for the self-energy at the two-loop order [4].

\section{Discussion}

The results announced herein may be useful in physical scenarios where the external fields are static and the temperature is high. In this case, a considerable simplification should occur, since, in the configuration space, the SZM identity implies that one may compute the static effective action using a much simpler space-time independent background field configuration as in the oneloop case [3]. The two-loop result indicates that the effective action of static gravitational fields 
may also be obtained in a closed form, from the condition that the external field is space-time independent, in the configuration space.

We also remark that the Feynman graph topologies which we have considered is sufficiently general to encompass a class of field theories with a finite or infinite number of vertices (like gravity in the weak field approximation). We are now investigating the SZM identity for all two-loop 1PI Green's functions.

\section{References}

[1] J. I. Kapusta, Finite Temperature Field Theory (Cambridge University Press, Cambridge, England, 1989).

[2] M. L. Bellac, Thermal Field Theory (Cambridge University Press, Cambridge, England, 1996).

[3] F. T. Brandt and J. B. Siqueira, Phys.Rev. D85, 067701 (2012).

[4] F. T. Brandt and J. B. Siqueira, arXiv:1208.6585 [hep-th] (to appear in Phys. Rev. D).

[5] A. Rebhan, Nucl. Phys. B351, 706 (1991).

[6] S. P. Alwis and N. Ohta, Phys. Rev. D52, 3529 (1995).

[7] J. Frenkel, S. H. Pereira, and N. Takahashi, Phys. Rev. D79, 085001 (2009). 